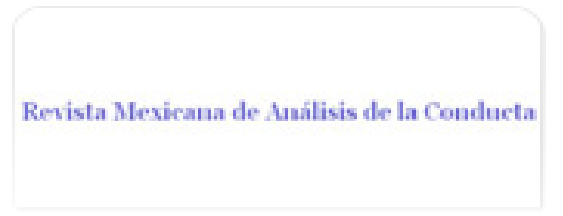

Revista Mexicana de Análisis de la Conducta ISSN: 0185-4534

editora@rmac-mx.org

Sociedad Mexicana de Análisis de la Conducta México

Guevara Benítez, Yolanda; Macotela Flores, Silvia

Evaluación del avance académico en alumnos de primer grado

Revista Mexicana de Análisis de la Conducta, vol. 32, núm. 2, diciembre, 2006, pp. 129-153

Sociedad Mexicana de Análisis de la Conducta

Guadalajara, México

Disponible en: http://www.redalyc.org/articulo.oa?id=59332204

- Cómo citar el artículo

- Número completo

- Más información del artículo

- Página de la revista en redalyc.org

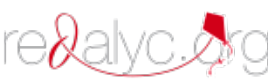

Sistema de Información Científica

Red de Revistas Científicas de América Latina, el Caribe, España y Portugal Proyecto académico sin fines de lucro, desarrollado bajo la iniciativa de acceso abierto 


\title{
EVALUACIÓN DEL AVANCE ACADÉMICO EN ALUMNOS DE PRIMER GRADO
}

\author{
ASSESSMENT OF THE ACADEMIC PERFORMANCE \\ OF FIRST GRADE STUDENTS \\ YOLANDA GUEVARA BENÍTEZ Y SILVIA MACOTELA FLORES \\ UNIVERSIDAD NACIONAL AUTÓNOMA DE MÉXICO
}

\begin{abstract}
RESUMEN
El objetivo del presente estudio fue realizar una evaluación del avance académico de alumnos de primer grado de primaria, en diversas habilidades de lectura, escritura y matemáticas, y contrastar la ejecución real de los alumnos, con los objetivos del programa oficial. Un objetivo adicional fue determinar la posible relación entre las ejecuciones de los niños y su historia académica previa (haber cursado o no preescolar). Los participantes fueron 20 alumnos de primer grado, pertenecientes a dos escuelas públicas de nivel socio-económico bajo, en el Estado de México. 10 niños con experiencia preescolar y 10 sin esta experiencia. Las mediciones, apoyadas en la evaluación referida a criterio y basada en el currículum, fueron aplicadas en cuatro períodos diferentes: una al inicio del año escolar, dos más en medio del ciclo, y una al final. Los resultados indican que los alumnos con experiencia preescolar tuvieron mejores ejecuciones que los niños sin preescolar en la medición inicial de las tres áreas académicas. Se constató que las diferencias entre las ejecuciones de los grupos tienden a disminuir a lo largo del ciclo escolar. Sin embargo, las ejecuciones finales indican que los objetivos del programa son sólo parcialmente cumplidos, en ambos grupos de niños. Se discute la falta de correspondencia entre las ejecuciones de los alumnos y las expectativas del programa, así como la necesidad de continuar investigando para determinar las variables relevantes que contribuyen a este fenómeno. Los resultados también son discutidos en relación a las ventajas del tipo de medición utilizada para el análisis de las ejecuciones de lectura, escritura y matemáticas.

Palabras clave: experiencia preescolar, lecto-escritura, matemáticas; medición con referencia a criterio; evaluación con referencia a currículum.
\end{abstract}




\begin{abstract}
The purpose of the present study was to contrast children's actual performance in reading, writing and mathematics, with the objectives of the official educational program. An additional purpose was to determine possible relations between performance and children's previous academic history (preschool vs. no preschool). Subjects were 20 first grade children belonging to two public schools of low SE level in the State of Mexico. 10 were children who had preschool experience and 10 lacked this experience. Assessment supported by criterion-referenced and curriculum-based measures was conducted in four different periods: one at the beginning of the school year, two more in the middle of the cycle and one at the end. Results indicate that children with preschool experience perform better than children without this experience in the initial assessment in the three academic areas. Differences in performance between groups tend to decrease along the school year. Nevertheless, actual performance indicated that program objectives are only accomplished partially, regardless of previous experience. The lack of correspondence between actual performance and program expectations is discussed in terms of the need to continue research in order to determine relevant variables that are contributing to this phenomenon. Results are also discussed in relation to advantages of the type of measures used for the analysis of reading, writing and mathematics performance.
\end{abstract}

Key Words: preschool experience; reading, writing and mathematics; criterion-referenced measurement, curriculum-based assessment.

El desarrollo teórico-metodológico del Análisis de la Conducta le ha permitido realizar contribuciones para la educación, principalmente en lo referente a las áreas de diseño curricular, evaluación de la educación, y manejo de factores relacionados con el bajo rendimiento escolar.

Como lo señalan López, Santoyo, Gómez y Aguilar (1995), la psicología conductual se preocupa por estudiar la forma en que los organismos interactúan con los objetos y eventos de su entorno, y en ese sentido es una teoría de la competencia. En el campo educativo, el Análisis de la Conducta requiere identificar el nivel de competencia esperado, el sistema normativo apropiado y los modos educativos por emplear. Su principal preocupación es desarrollar tecnología para la enseñanza de competencias académicas específicas, tomando como base la evaluación y la enseñanza de tareas académicas con diferentes grados de dificultad.

Todo ello conduce a lo que se considera como el legado más importante de esta corriente a la educación: la sistematización. “...el conductismo ha centrado la atención en las condiciones que permiten que el individuo apre- 
nda; esto permite instrumentar situaciones de enseñanza-aprendizaje y analizar las condiciones concretas del proceso" (Ribes, en Glazman, Figueroa, Follari y Rodríguez, 1983, p. 281). La teoría conductual ha hecho diversas aportaciones educativas relacionadas con los "esfuerzos sistemáticos para influir en la conducta de los estudiantes de manera eficiente, consistente y positiva... aplicando la ciencia a la conducta humana" (Biehler y Snowman, 1992, p. 169).

Las aportaciones del modelo conductual al currículum se pueden resumir en los siguientes aspectos: 1 . La traducción de principios generales del aprendizaje y formación de conceptos, a procedimientos de enseñanza. 2. El desarrollo de formas para facilitar el aprendizaje humano a través del desarrollo curricular que considera lo general y lo específico, y que va de lo simple a lo complejo. 3. El desarrollo de una tecnología educativa que permite la sistematización de la enseñanza, consistente en la selección y definición de objetivos, el análisis de tareas, el diseño de instrumentos de evaluación basados en objetivos instruccionales específicos y secuenciados, y la interrelación de factores: objetivos, materiales, métodos y procedimientos, medición de progresos, evaluación de logros y errores de los programas (Bannan y Grabowsky, 1995; Becker, 1992; Bender, 1992; Hannafin y Freeman, 1995; Herman y Herman, 1995; Howell, Fox y Morehead, 1993; Kaufman y Swart, 1995; Salvia y Hughes, 1990; Spotts y Bowman, 1995; Strommen, 1992).

En lo que respecta a la evaluación, el Análisis de la Conducta sostiene que se trata de un proceso continuo e inseparable de la enseñanza, por ello se plantea la necesidad de evaluar los repertorios conductuales con que los alumnos inician su instrucción, los avances que van logrando con un programa de instrucción determinado, y la consecución de los objetivos curriculares. Este proceso de evaluación permite no sólo observar los avances en el desarrollo de las habilidades de conducta que son los objetivos del programa, sino detectar los tipos de error que son frecuentes en los alumnos que se someten a dicho programa y por lo tanto evaluar su eficacia, en términos de los aciertos y errores de los alumnos y en términos de la congruencia que guarda con respecto al nivel con que los alumnos se incorporan en él.

En tal orden de ideas, los aportes de la psicología conductual a la evaluación en la educación están asociados fundamentalmente al diseño de herramientas que dan cuenta del desempeño en diferentes dominios así como de los logros obtenidos a través de la instrucción. Es así que, como alternativa a la evaluación con referencia a la norma, este enfoque ofrece lo que se conoce como evaluación para la enseñanza sustentada por la medición con referencia a criterio.

Macotela, Bermúdez y Castañeda (1995) explican que la medición con referencia a la norma se refiere a la aplicación de pruebas estandarizadas, desarrolladas bajo los principios de la psicometría; la información que proporcionan dichas pruebas es de naturaleza cuantitativa, lo que permite comparar las eje- 
cuciones de cada niño contra un parámetro o estándar establecido con una población específica. Estas autoras afirman "Cuando se trata de ayudar al maestro en la planeación de programas instruccionales, se recomienda utilizar pruebas basadas en las medidas con referencia a criterio, más que en las referidas a la norma. Es evidente que a nivel educacional, es más importante preocuparse por identificar las habilidades que posee el alumno y aquellas de las cuales carece, que compararlo con otros" (Macotela y cols., 1995, p. 11). También aclaran que las medidas con referencia a criterio han sido desarrolladas dentro de la óptica conductual como herramientas para medir el desarrollo de habilidades en términos de destrezas específicas.

En dicha aproximación a la evaluación cobra particular importancia una estrategia para organizar las destrezas conductuales: el análisis de tareas. Este modelo señala que para el logro de metas, tanto de la evaluación como de la enseñanza, deben realizarse análisis de tareas específicas y de objetivos instruccionales definidos; asimismo, las estrategias de intervención están diseñadas para el entrenamiento de subhabilidades (objetivos específicos) que lleven paso a paso a la obtención de habilidades generales, denominados objetivos complejos o a largo plazo. De acuerdo con Howell, Kaplan y O'Connel (1979) el modelo de análisis de tareas constituye una de las más importantes contribuciones de la psicología conductual a la educación. Incluso el modelo ha sido el antecedente directo de lo que hoy se conoce como análisis cognoscitivo de tareas (Dockrell y McShane, 1993). Derivada de las medidas con referencia a criterio se encuentra la denominada evaluación basada en currículo, la cual articula la relación entre evaluación, currículum e instrucción de la manera siguiente: el currículum es aquello que se enseña, la instrucción es la forma como se enseña, y la evaluación es la estrategia que guía y coordina el proceso completo. Este enfoque plantea que la evaluación debe incluir análisis de conceptos, análisis de tareas y análisis de errores (Hallahan y Kauffman, 1991; Howell y cols., 1993).

Autores como Adelman y Taylor (1993), Hallahan, Kauffman y Lloyd (1999) y Macotela (1993), han enfocado sus esfuerzos a definir y estudiar sistemáticamente las razones por las cuales puede darse el fracaso escolar. De los planteamientos de dichos autores se conoce que en el bajo rendimiento escolar influyen diversos factores individuales, familiares y escolares. El modelo conductual ha hecho énfasis en la relación que existe entre las habilidades preacadémicas y el éxito en el desarrollo de la lectura y la escritura (Baker, Mackler, Sonnenschein y Serpell, 2001; Bowey, 1995; Buckner, Bassuk y Weinreb, 2001; Carroll, Snowling, Hulme y Stevenson, 2003). De hecho, Wallace, Larsen y Elksnin (1992) utilizan aprestamiento para la instrucción escolar como un término genérico que incluye habilidades cognoscitivas, sociales y de lenguaje que se requieren para un aprendizaje escolar eficiente; señalan que no puede decirse que un niño esté preparado para enfrentar la instrucción esco- 
lar sin niveles aceptables de dichas habilidades. Tales planteamientos guardan una estrecha relación con los hallazgos de la investigación psicológica acerca de la secuencia que sigue el desarrollo académico inicial, así como de los factores que influyen dicho desarrollo (Adams, Treiman y Pressley, 1998; Martínez, Martínez y Pérez, 2004).

Estudios recientes, llevados a cabo con amplias poblaciones de alumnos de diversos países, prueban que cuando los niños no han desarrollado niveles adecuados de conducta preacadémica y lingüística su adquisición de la lecto-escritura se dificulta y puede llegar a presentarse fracaso escolar, especialmente durante los primeros años escolares (DiLalla, Marcus y Wright-Phillips, 2004; Guevara y Macotela, 2005; Leppänen, Niemi, Aunola y Nurmi, 2004). También se ha probado ampliamente que el desarrollo de tales habilidades se ve afectado por el nivel socioeconómico, intelectual y educativo de las familias (Muter, Hulme, Snowling y Stevenson, 2004; Poe, Burchinal y Roberts, 2004).

Tomando como base los planteamientos psicológicos mencionados, dentro de la literatura se encuentra un creciente interés por analizar las características que debe tener el currículum de las escuelas públicas para integrar a todos los niños, propiciando el logro de objetivos académicos y evitando los problemas de fracaso escolar (Dearing, McCartney, Weiss, Kreider y Simpkins, 2004; Guevara y Macotela, 2005; Guevara, 2006).

En países como Estados Unidos, la investigación realizada en el campo se traduce en la aplicación de una serie de políticas educativas y recomendaciones dirigidas al personal de las escuelas públicas, para detectar estudiantes de "alto riesgo" (tomando en cuenta la estructura social, familiar, escolar y las características personales de los alumnos), así como para poner en práctica estrategias educativas que ayuden a prevenir o remediar los retrasos escolares de los niños (De Jong y Leseman, 2001; González, 2004; Morrison, Rimm-Kauffman y Pianta, 2003; Muter, Hulme, Snowling y Stevenson, 2004; Poe, Burchinal y Roberts, 2004; Salsa y Peralta, 2001; Vacha y McLaughlin, 1992). También se han modificado objetivos, estructuras y sistemas de motivación dentro del salón de clases para evitar el fracaso escolar de niños que no cumplen con habilidades básicas preacadémicas; estos niños son sometidos a programas compensatorios para que desarrollen habilidades que les permitan enfrentarse al programa regular. Desafortunadamente, en México no se cuenta con un sistema de evaluación integrado al currículum (como ya lo señalaron Martínez, Cepeda, Fuentes y Burgos desde 1995), lo cual hace necesario realizar estudios enfocados a conocer diversos aspectos del proceso de enseñanza-aprendizaje, cuyos datos nos permitan hacer sugerencias educativas aplicables a nuestro país. Algunos avances al respecto han sido reportados por Guevara y Macotela (2005).

El presente estudio retoma los tres aspectos que se han descrito antes como aportaciones del Análisis de la Conducta a la educación, y especialmente 
de la evaluación referida a criterio y basada en el currículum. El objetivo del trabajo fue realizar una evaluación del avance académico de niños de primer grado de primaria, en diferentes habilidades de lectura, escritura y matemáticas, con especial énfasis en el análisis de los logros y los errores de los alumnos en distintos momentos, a lo largo del ciclo escolar. Adicionalmente, se buscó ubicar posibles relaciones entre las ejecuciones académica de los niños y su historial educativo (haber cursado o no el nivel preescolar), así como contrastar las habilidades académicas de los alumnos con los objetivos del programa oficial.

\section{MÉTODO}

\section{Participantes}

Participaron 20 niños de reciente ingreso al primer grado de primaria, pertenecientes a dos escuelas públicas del Estado de México. Fueron elegidos con los siguientes criterios: 1) que al inicio del ciclo escolar no supieran leer ni sílabas, ni palabras de manera convencional; 2) que pertenecieran a escuelas públicas de la zona metropolitana del Estado de México, ubicadas en zonas poblacionales de clase media-baja (colonias proletarias); 3 ) diez de los niños ingresaron a primer grado habiendo cursado educación preescolar (Grupo 1 en este estudio), y diez niños ingresaron a primer grado sin dicho curso propedéutico (Grupo 2).

\section{Procedimiento}

Se pidió la colaboración de directores y profesores de las escuelas primarias para que proporcionaran datos respecto a los alumnos que ingresaron a primer grado habiendo cursado el nivel preescolar y los que no cursaron dicho nivel previo. También se pidió su autorización para la aplicación de cuatro evaluaciones a lo largo del ciclo escolar, para hacer un seguimiento académico de los alumnos. Las evaluaciones se realizaron en las aulas que las propias escuelas proporcionaron. Los niños fueron evaluados de manera individual en todos los casos, en un salón de clases, de aproximadamente $3 \times 3$ metros, que contaba con pizarrón y mesabancos. Al comienzo de la sesión, el evaluador anotaba la hora de inicio y daba al alumno las instrucciones generales para dar respuesta a la prueba; después le daba la instrucción correspondiente a cada reactivo, presentando el material; esperaba a que el niño diera la respuesta a la pregunta o a que realizara la operación para registrar si la respuesta fue correcta o incorrecta. Una vez concluida la aplicación de la prueba, se anotaba la hora de finalización y las observaciones respectivas.

Cuando se concluyó la evaluación de todos los alumnos se procedió a 
la calificación de cada prueba, con base en los formatos de registro y en las hojas con las respuestas de los niños. Con base en los datos obtenidos se compararon las ejecuciones de los alumnos de un grupo y otro (con y sin preescolar), en diferentes momentos del ciclo escolar.

\section{Instrumentos}

Para llevar a cabo el presente estudio se utilizó el instrumento denominado Inventario de Ejecución Académica (IDEA), de Macotela, Bermúdez y Castañeda (1991), dado que cumple con los requisitos de la evaluación referida a criterio: 1) mide directamente la ejecución del alumno, en términos de conducta observable; 2) evalúa conductas relacionadas directamente con los aspectos que se pretende evaluar; 3) permite evaluar los cambios en la conducta al ser aplicada en dos o más momentos diferentes; 4) permite ubicar cuáles habilidades conductuales ha desarrollado cada niño y aquéllas de las cuales carece, y 5) su interés principal no es comparar a un individuo en particular con respecto a una norma poblacional, sino observar el propio avance del alumno. Este instrumento cumple con dos criterios adicionales, derivados de la evaluación basada en el currículum: evalúa conductas relacionadas directamente con los programas educativos y está estructurado a partir de un análisis de tareas, lo que permite identificar las habilidades desarrolladas por el alumno y las que aún no desarrolla. Fue diseñado para evaluar las habilidades de lectura, escritura y matemáticas en alumnos de primero, segundo y tercer grados de primaria, y fue validado con los criterios psicométricos correspondientes, para su uso en poblaciones mexicanas; en los trabajos de Maldonado (1999) y Martínez (2002) se reportan sus propiedades técnicas de validez y confiabilidad. EI IDEA permite evaluar la ejecución de las áreas académicas prioritarias, a través de datos cuantitativos (registro de puntajes en cada prueba) y cualitativos (derivados de la observación y del análisis de productos permanentes de las ejecuciones de los niños). En el presente estudio se utilizó la parte del instrumento correspondiente al primer grado de educación primaria, en donde se evalúan:

Lectura: incluye lectura en silencio y comprensión (señalando dibujos representativos) de números, palabras y enunciados, así como lectura oral y comprensión (señalando dibujos representativos) de palabras, enunciados y un cuento corto, además se incluyen preguntas de comprensión del cuento. Total de reactivos 45 .

Escritura: copia y comprensión (señalando dibujos representativos) de números, palabras y enunciados, así como el dictado y comprensión (señalando dibujos representativos) de números, palabras y enunciados. Total de reactivos 23.

Matemáticas: contar figuras, ubicar cuál conjunto de figuras es mayor o menor, ubicar las cifras correspondientes a un conjunto de figuras, encerrar en 
un círculo cierto número de unidades y decenas en conjuntos de figuras, identificar signos de suma y resta, sumar con figuras, restar con figuras, ubicar fracciones en figuras y en números escritos, marcar en una cifra escrita el número correspondiente a las unidades o a las decenas, realizar sumas escritas de un dígito y de dos dígitos y solución de problemas que implican suma y resta. Total de reactivos 46 .

Para obtener datos adicionales relativos a la lecto-escritura de los alumnos, en los mismos momentos en que se aplicó el IDEA se aplicaron pruebas cortas de administración frecuente, consistentes en presentar a los niños diez palabras escritas para que las leyeran y realizaran con ellas una composición escrita; las palabras fueron: casa, sol, silla, gato, flor, cama, perro, tele, mesa y lápiz. Adicionalmente se realizó el análisis del cuaderno de cada alumno para observar la calidad de su escritura, en los mismos cuatro momentos de evaluación.

\section{Materiales}

Instrumento IDEA, lápices y cuadernos.

\section{Criterios de análisis}

Se consideraron las respuestas que los alumnos dieron a cada reactivo del instrumento IDEA, con base en los criterios que estipula para respuestas correctas e incorrectas. Además de los datos cuantitativos del instrumento, el avance académico de los niños se evaluó analizando: 1) los productos permanentes de las evaluaciones del IDEA (hojas de respuesta), 2) los resultados de las pruebas cortas de administración frecuente donde cada alumno debía leer 10 palabras bisílabas y realizar con ellas una composición escrita, y 3) el análisis de la escritura que los alumnos hacían en sus cuadernos escolares.

\section{RESULTADOS}

Para ordenar y presentar los datos cuantitativos del IDEA, se sumaron los puntajes obtenidos por cada alumno en las tres áreas académicas durante la última evaluación, al final del ciclo escolar. Los sujetos del Grupo 1, que ingresaron con preescolar, fueron numerados del 1 al 10 y presentados en orden descendente, mientras que los sujetos del Grupo 2, que ingresaron sin preescolar, fueron numerados del 11 al 20, también en orden descendente, de acuerdo a sus calificaciones globales finales en el IDEA.

La Tabla 1 corresponde al Grupo 1 (sujetos con preescolar) y muestra el puntaje obtenido por cada uno de los alumnos, en las cuatro aplicaciones del 
instrumento, para las tres áreas académicas: lectura, escritura y matemáticas. La Tabla 2 muestra los resultados de los sujetos del Grupo 2. Al observar las tablas puede apreciarse que los alumnos del grupo con preescolar obtuvieron puntajes finales globales dentro de un rango que va de las 59 a las 100 respuestas correctas, del total de 114 posibles ( 45 de lectura, 23 de escritura y 46 de matemáticas), mientras los sujetos sin preescolar obtuvieron puntajes finales globales dentro de un rango de 36 a 94 respuestas correctas. Ocho alumnos del Grupo 1 obtuvieron calificaciones de 80 puntos o más, mientras sólo tres alumnos del Grupo 2 alcanzaron dicho nivel. En contraparte, sólo un alumno del Grupo 1 obtuvo una calificación por debajo de 60 aciertos, mientras en el Grupo 2 cuatro alumnos estuvieron en ese caso.

Tabla 1: Muestra el número de aciertos obtenidos por cada uno de los 10 alumnos que ingresaron a primaria con preescolar, en las pruebas de lectura, escritura y matemáticas, en las cuatro aplicaciones del IDEA. La última columna muestra el número total de aciertos obtenido por cada alumno al final del ciclo, considerando las tres áreas académicas. El número de cada sujeto de este Grupo 1 fue asignado (del 1 al 10) de acuerdo a dicha calificación global final, en orden descendente.

\begin{tabular}{|c|c|c|c|c|c|c|c|c|c|c|c|c|c|}
\hline \multirow{3}{*}{$\begin{array}{l}\text { No. } \\
\text { participante }\end{array}$} & \multicolumn{12}{|c|}{ Inventario de Ejecución Académica } & \multirow{3}{*}{ Total } \\
\hline & \multicolumn{4}{|c|}{ Lectura } & \multicolumn{4}{|c|}{ Escritura } & \multicolumn{4}{|c|}{ Matemáticas } & \\
\hline & 1ra & $2 \mathrm{da}$ & $3 \mathrm{ra}$ & $4 \mathrm{ta}$ & 1ra & $2 \mathrm{da}$ & 3 ra & $4 \mathrm{ta}$ & 1ra & $2 \mathrm{da}$ & 3 ra & $4 \mathrm{ta}$ & \\
\hline Sujeto 1 & 6 & 35 & 36 & 44 & 5 & 21 & 23 & 23 & 18 & 27 & 33 & 33 & 100 \\
\hline Sujeto 2 & 5 & 31 & 34 & 41 & 5 & 15 & 21 & 22 & 12 & 28 & 30 & 34 & 97 \\
\hline Sujeto 3 & 7 & 31 & 35 & 36 & 4 & 17 & 23 & 23 & 12 & 28 & 34 & 36 & 95 \\
\hline Sujeto 4 & 1 & 8 & 31 & 34 & 2 & 2 & 23 & 23 & 11 & 14 & 19 & 35 & 92 \\
\hline Sujeto 5 & 6 & 30 & 36 & 38 & 5 & 11 & 20 & 20 & 19 & 28 & 32 & 33 & 91 \\
\hline Sujeto 6 & 6 & 15 & 36 & 36 & 2 & 11 & 15 & 21 & 11 & 28 & 31 & 34 & 91 \\
\hline Sujeto 7 & 2 & 20 & 32 & 35 & 0 & 6 & 23 & 23 & 13 & 15 & 27 & 32 & 90 \\
\hline Sujeto 8 & 5 & 16 & 17 & 29 & 6 & 8 & 9 & 17 & 22 & 25 & 27 & 34 & 80 \\
\hline Sujeto 9 & 1 & 9 & 18 & 29 & 1 & 1 & 6 & 11 & 10 & 19 & 27 & 31 & 71 \\
\hline Sujeto 10 & 5 & 15 & 15 & 23 & 2 & 6 & 11 & 15 & 9 & 17 & 17 & 21 & 59 \\
\hline
\end{tabular}

Total posible de lectura 45

Total posible de escritura 23

Total posible de matemáticas 46 
Tabla 2. Muestra el número de aciertos obtenidos por cada uno de los 10 alumnos que ingresaron a primaria sin preescolar, en las pruebas de lectura, escritura y matemáticas, en las cuatro aplicaciones del IDEA. La última columna muestra el número total de aciertos obtenido por cada alumno al final del ciclo, considerando las tres áreas académicas. El número de cada sujeto de este Grupo 2 fue asignado (del 11 al 20) de acuerdo a dicha calificación global final, en orden descendente.

\begin{tabular}{|c|c|c|c|c|c|c|c|c|c|c|c|c|c|}
\hline \multirow{3}{*}{$\begin{array}{l}\text { No. } \\
\text { participante }\end{array}$} & \multicolumn{12}{|c|}{ Inventario de Ejecución Académica } & \multirow{3}{*}{ Total } \\
\hline & \multicolumn{4}{|c|}{ Lectura } & \multicolumn{4}{|c|}{ Escritura } & \multicolumn{4}{|c|}{ Matemáticas } & \\
\hline & 1ra & 2da & $3 \mathrm{ra}$ & 4 ta & 1ra & 2da & 3ra & 4 ta & 1ra & $2 \mathrm{da}$ & 3ra & 4ta & \\
\hline Sujeto 11 & 0 & 19 & 32 & 35 & 0 & 9 & 18 & 21 & 5 & 24 & 35 & 38 & 94 \\
\hline Sujeto 12 & 12 & 14 & 33 & 33 & 2 & 10 & 21 & 23 & 14 & 26 & 30 & 30 & 86 \\
\hline Sujeto 13 & 0 & 10 & 30 & 33 & 5 & 5 & 20 & 23 & 10 & 25 & 27 & 30 & 86 \\
\hline Sujeto 14 & 4 & 18 & 33 & 34 & 6 & 9 & 22 & 23 & 9 & 17 & 19 & 22 & 79 \\
\hline Sujeto 15 & 4 & 8 & 23 & 31 & 1 & 2 & 14 & 18 & 7 & 12 & 20 & 24 & 73 \\
\hline Sujeto 16 & 5 & 10 & 27 & 35 & 3 & 3 & 9 & 14 & 9 & 15 & 20 & 22 & 71 \\
\hline Sujeto 17 & 2 & 5 & 21 & 24 & 0 & 2 & 9 & 13 & 5 & 13 & 19 & 21 & 58 \\
\hline Sujeto 18 & 0 & 4 & 13 & 20 & 1 & 2 & 4 & 8 & 0 & 10 & 15 & 29 & 57 \\
\hline Sujeto 19 & 5 & 8 & 12 & 16 & 1 & 1 & 3 & 3 & 14 & 20 & 21 & 27 & 46 \\
\hline Sujeto 20 & 1 & 8 & 10 & 10 & 1 & 1 & 7 & 7 & 7 & 11 & 11 & 19 & 36 \\
\hline
\end{tabular}

Total posible de lectura 45

Total posible de escritura 23

Total posible de matemáticas 46

Para estar en posibilidad de comparar los resultados obtenidos por los alumnos en las diferentes áreas académicas, los puntajes fueron traducidos a porcentajes de respuestas correctas y con tales datos se elaboraron las Figuras 1 y 2.

En la Figura 1 puede observarse el avance académico de cada uno de los 10 alumnos con preescolar, a través de los porcentajes de ejecución en lectura, escritura y matemáticas. Se aprecia que todos los alumnos de este Grupo 1 mostraron avances sostenidos a lo largo del ciclo escolar en las tres áreas académicas, aunque cabe resaltar que los mayores avances se presentaron en lectura y escritura. En el área de lectura todos los sujetos mostraron niveles iniciales por debajo del $20 \%$ de respuestas correctas; al observar las ejecuciones de los Sujetos 1, 2, 3 y 5 se puede apreciar que estos alumnos mostraron un avance de alrededor de 50 puntos porcentuales durante los primeros tres meses del curso, y que para la tercera y cuarta evaluaciones sus avances fueron menores; mientras los Sujetos 4,6 y 7 mostraron pocos avances en el primer trimestre del curso (manteniendo ejecuciones por deba- 

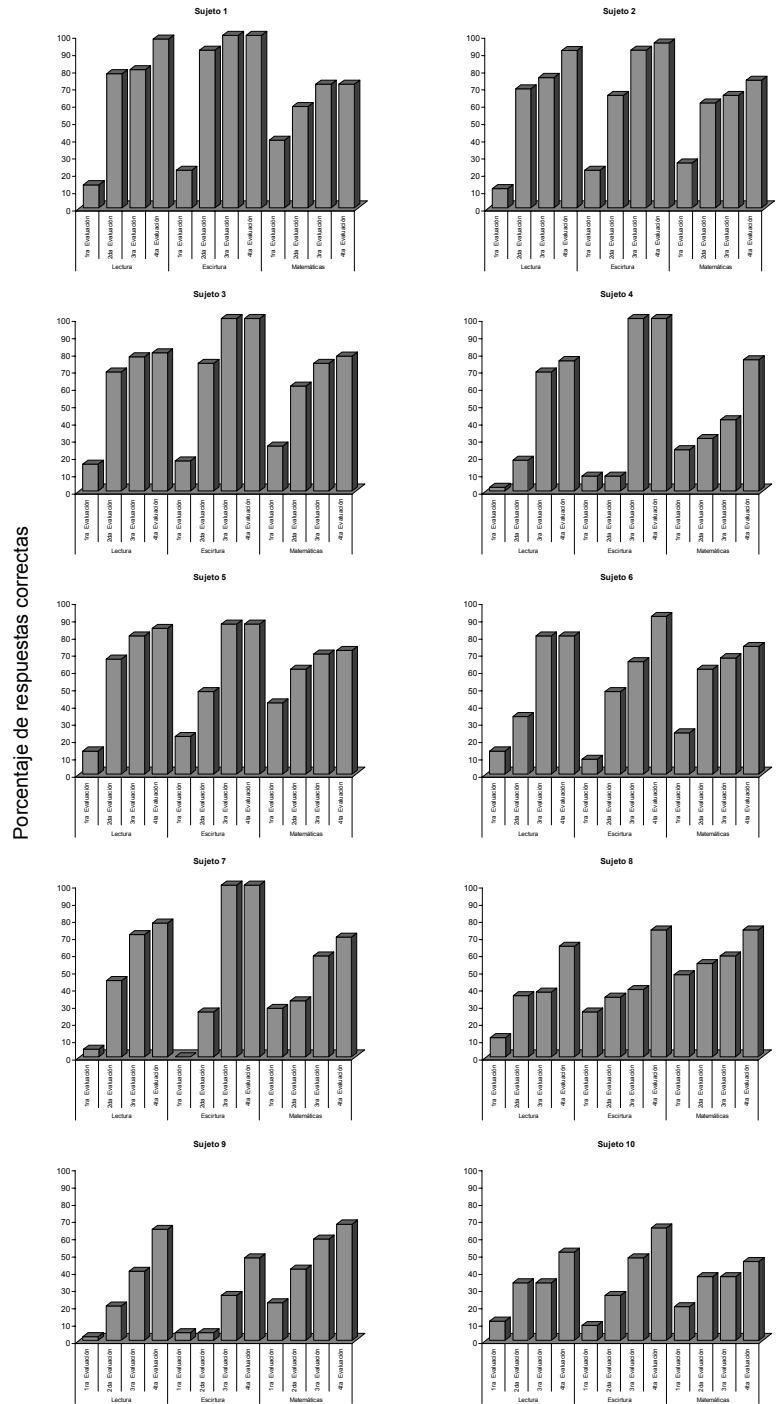

Subpruebas y Evaluaciones

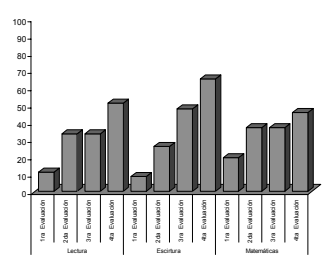

Subpruebas y Evaluaciones

Figura 1: Muestra el avance académico de cada uno de los 10 alumnos del Grupo 1 (que ingresaron a primaria con preescolar), a través de sus porcentajes de ejecución en cada una de las cuatro evaluaciones de lectura, escritura y matemáticas. 


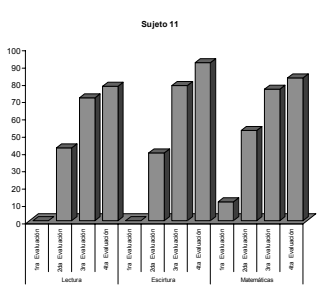

sujet 13

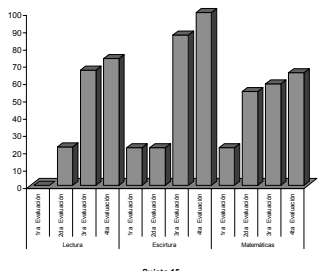

0
0
0
0
0
0
0
0
$\mathbb{0}$
0
0
0
0
0
0
0
0
0
0
0
0
0
0
0
0
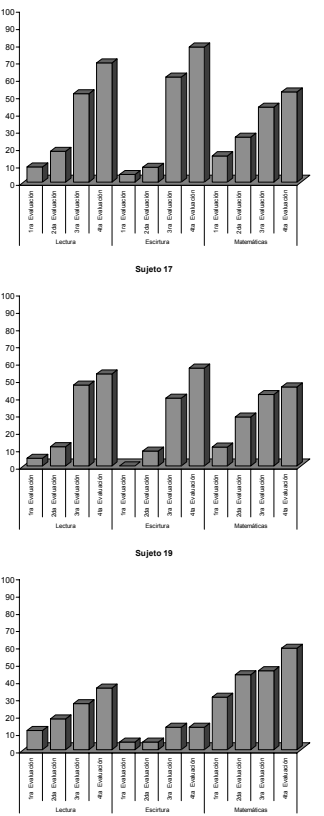

Subpruebas y Evaluaciones

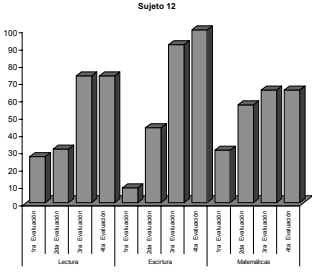

Sujeto 14
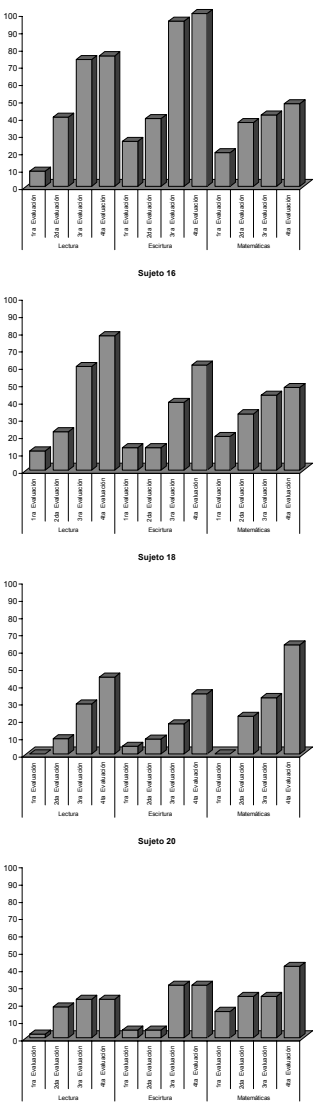

Subpruebas y Evaluaciones

Figura 2: Muestra el avance académico de cada uno de los 10 alumnos del Grupo 2 (que ingresaron a primaria sin preescolar), a través de sus porcentajes de ejecución en cada una de las cuatro evaluaciones de lectura, escritura y matemáticas. 
jo del $50 \%$ ) y una mayor ganancia en el periodo comprendido entre el cuarto y el sexto mes (hasta alcanzar niveles cercanos al $80 \%$ ). Estos siete sujetos fueron quienes lograron mejores ejecuciones finales -entre 80 y $100 \%$ de respuestas correctas-, mientras los Sujetos 9 y 10 mostraron menores niveles en esta área académica a lo largo de todo el ciclo escolar y para la evaluación final alcanzaron entre el 50 y el $64 \%$ de respuestas correctas. Los niveles iniciales y los avances en escritura fueron muy similares a los de lectura en la mayoría de los sujetos de este Grupo 1 . En cambio en matemáticas se observa un fenómeno muy distinto: los porcentajes iniciales de esta área fueron los más altos -entre el 20 y el $40 \%$ - en los 10 sujetos de este grupo, y aunque se aprecian cambios de una a otra evaluación, para el final del ciclo escolar ninguno de los 10 sujetos alcanzó el $80 \%$ de respuestas correctas, nueve de ellos obtuvieron entre 67 y $78 \%$ y el sujeto 10 alcanzó $45 \%$.

En la Figura 2 puede observarse el avance académico de cada uno de los 10 alumnos que ingresaron sin preescolar, a través de los porcentajes de ejecución en lectura, escritura y matemáticas. Se aprecia que, a excepción del sujeto 20, los alumnos de este Grupo 2 mostraron avances sostenidos a lo largo del ciclo escolar en las tres áreas académicas, aunque el nivel alcanzado, en general, es menor al de los alumnos del Grupo 1. En el área de lectura el sujeto 12 obtuvo un nivel inicial de $26 \%$, ligeramente mayor al obtenido por los alumnos con preescolar, pero los otros nueve sujetos mostraron niveles cercanos a cero. Para la segunda evaluación, los sujetos 11,12 y 14 obtuvieron entre el 30 y el $40 \%$, pero los otros siete sujetos se mantuvieron entre 10 y $30 \%$ de respuestas correctas. Para la tercera evaluación, cuatro de estos alumnos (sujetos 11 al 14) alcanzaron niveles entre 70 y $80 \%$ de ejecución, mientras los sujetos 15,16 y 17 logran un nivel alrededor del $50 \%$ y los últimos tres sujetos continuaron con ejecuciones cercanas al $30 \%$. En la evaluación final, ninguno de los alumnos del Grupo 2 llegó al 80\% de ejecución en lectura, de hecho, los últimos cuatro sujetos mantuvieron porcentajes menores al $60 \%$. En escritura los niveles iniciales y de segunda evaluación fueron similares a los descritos para el caso de la lectura, pero a partir de la tercera evaluación se observan avances sustanciales en escritura en siete de los sujetos. Para la evaluación final, cuatro de los alumnos (sujetos 11 a 14) obtuvieron porcentajes entre el 90 y el $100 \%$, que se asemejan a los logrados por la mayoría de los sujetos del Grupo 1, aunque los últimos cuatro sujetos mantuvieron porcentajes menores al $60 \%$, tal como ocurrió en su propia ejecución de la lectura. En matemáticas se observa que, tal como ocurrió para el Grupo 1, los porcentajes iniciales de esta área fueron los más altos, aunque estos niños obtuvieron en promedio 10 puntos porcentuales menos que los niños con preescolar -en este caso las ejecuciones estuvieron entre el 10 y el 30\%- en 9 de los 10 sujetos de este grupo. Se aprecian cambios sucesivos en todos los estudiantes y para el final del ciclo escolar el 
sujeto 11 logra el $83 \%$ de ejecución, la mayor en matemáticas de todos los alumnos evaluados, pero los restantes nueve sujetos obtuvieron entre 41 y $65 \%$ de respuestas correctas.

Para presentar un panorama más detallado, en términos del desarrollo de destrezas específicas, a continuación se exponen los avances y errores detectados en cada momento de evaluación por los alumnos de ambos grupos.

\section{Análisis del avance en Lectura}

Durante la primera evaluación (al inicio del ciclo escolar) la lectura de palabras no es realizada por los niños de ningún grupo, aunque algunos del Grupo 1, con preescolar, pudieron identificar ciertas letras. Respecto a la lectura de números, sí se observaron diferencias entre los participantes. En el Grupo 1, siete alumnos leyeron números de una y dos cifras (sujetos 1, 2, 3, 5, 6, 8 y 10), uno leyó números de una cifra (sujeto 7 ) y dos alumnos (sujetos 4 y 9 ) no leyeron ningún número; mientras que en el Grupo 2, sólo tres niños leyeron números de una y dos cifras (sujetos 12, 16 y 19), tres leyeron de una cifra $(14,15$ y 17) y cuatro no supieron leer ningún número. Para la segunda evaluación de lectura con el instrumento IDEA, ocho participantes del Grupo 1 leyeron números de una y dos cifras (los sujetos 4 y 9 continuaron sin hacerlo), mientras que en el Grupo 2 realizaron esta tarea seis sujetos $(11,12,13,14,16$ y 19). Cuando se pidió a los niños leer palabras específicas de dos y tres sílabas, seis alumnos del Grupo 1 (sujetos 1, 2, 3, 5, 6, 7), así como tres alumnos del Grupo 2 (11, 12 y 14), mostraron avances sustanciales en la identificación de los dibujos representativos de palabras leídas en silencio, y en la lectura oral de palabras señalando los dibujos representativos; cabe hacer la observación de que su lectura era realizada letra por letra, o sílaba por sílaba, lo que implicó varios minutos para leer cada palabra; también se observó que frecuentemente se equivocaban de letra, pero eran capaces de auto-corregirse; lo que se les dificultó mucho fue la lectura de sílabas compuestas ( $c r, g r, p r)$, sólo podían leer sílabas directas. Los otros cuatro alumnos del Grupo 1 y siete del Grupo 2 mostraron sólo aproximaciones, leyendo algunas letras o sílabas directas de las palabras presentadas y cometieron frecuentes errores de sustitución y omisión de letras, sin auto-corregirse.

Para la tercera aplicación de la prueba (casi siete meses después de iniciado el curso escolar) todos los participantes del Grupo 1 leyeron números de una y dos cifras, mientras que en el Grupo 2 lo hicieron siete (sujetos del 11 al 17). La lectura en silencio -y sus correspondientes pruebas de comprensión- para el caso de palabras, fue realizada por la mayoría de los alumnos del Grupo 1 (a excepción de los sujetos 8, 9 y 10) y por la mitad de los niños del Grupo 2 (sujetos 11, 12, 13, 14 y 16); para el caso de enunciados, por seis sujetos $(1,2$, $3,5,6$ y 7$)$ del Grupo 1 y tres sujetos $(11,12$ y 14$)$ del Grupo 2. La lectura oral 
señalando los dibujos representativos mostró mayores diferencias entre grupos. En el caso de palabras, fue realizada por siete alumnos del Grupo 1 (con las mismas excepciones antes mencionadas) y por tres del Grupo 2 (11, 12 y 14); la lectura oral de enunciados la realizaron cinco sujetos $(1,2,3,5$ y 6$)$ del Grupo 1 y del Grupo 2 sólo el sujeto 14. La lectura de un cuento señalando los dibujos representativos fue concluida por cuatro alumnos de cada grupo (sujetos 1,3 , $5,6,11,12,13$ y 14), y en este caso las diferencias se relacionaron principalmente con los problemas de fluidez, mayores en los alumnos del Grupo 2. Pudo observarse también que, aun cuando estos ocho niños lograron leer el cuento y señalar sus dibujos representativos, sólo un alumno (sujeto 1) fue capaz de contestar las preguntas de contenido relativas al cuento leído.

La evaluación final mostró cómo concluyeron los alumnos el ciclo escolar: Lectura en silencio y comprensión. En números, sólo falló el sujeto 20 , que continuaba leyendo únicamente una cifra. En lo que respecta a señalar palabras, se equivocaron solamente el sujeto 10 y el 20 . En lo relativo a señalar enunciados, cometieron errores el sujeto 10, del Grupo 1 y los sujetos 18 , 19 y 20 , del Grupo 2 . En señalar dibujos que representan la palabra leída, se equivocaron dos (sujetos 9 y 10) y tres alumnos (sujetos 18, 19 y 20), respectivamente. En señalar dibujos que representan enunciados, se equivocaron los mismos alumnos que en el caso de palabras, así como los sujetos 15 y 17). En Lectura oral y comprensión se observó que sólo se equivocaron los sujetos 9 y 10 del Grupo 1 y los sujetos 15, 17, 18, 19 y 20 del Grupo 2, para el caso de palabras. La lectura oral de enunciados fue realizada por siete alumnos del grupo con preescolar (cometiendo errores los sujetos 8,9 y 10), y por tres alumnos del grupo sin preescolar (sujetos 11, 12 y 14). Los mismos siete alumnos del Grupo 1 y cuatro del Grupo 2 (agregándose el sujeto $13)$, realizaron la lectura oral del cuento. Sin embargo, sólo tres alumnos del Grupo 1 (sujetos 1, 2, y 5) y uno (11) del Grupo 2, contestaron correctamente las preguntas relativas al cuento leído.

Cabe hacer la observación de que, al final del año escolar, los alumnos presentaron todavía problemas de fluidez, discriminación y comprensión de la lectura, que la mayoría de los niños leían cada palabra lentamente, y que tales problemas fueron mayores en los niños del Grupo 2.

\section{Análisis del avance en Escritura}

Como antes se mencionó, los niveles iniciales y el avance en escritura guardaron concordancia con los obtenidos en el área de lectura, en la mayoría de los alumnos evaluados. Durante la primera evaluación con el IDEA, prácticamente todos los niños del Grupo 1 fueron capaces de copiar números y palabras (exceptuando a los sujetos 7 y 9 ), y cinco niños copiaron enunciados (sujetos 1, 2, 3, 5 y 8); en el Grupo 2, cuatro niños (sujetos 12, 13, 14 y 
16) copiaron números y palabras, y sólo dos (13 y 14) copiaron enunciados. Todos los alumnos con preescolar presentaron problemas de tamaño de las letras (muy grandes, muy pequeñas o combinando tamaños) y problemas de calidad de líneas (muy marcadas o muy tenues); algunos mostraron además problemas de sustitución o distorsión de letras, aunque los problemas de distribución de espacios fueron pocos. En el caso de los alumnos sin preescolar, el sujeto 13 presentó problemas en la calidad de líneas y tamaño de letras, los otros nueve niños presentaron todos los problemas considerados en el análisis de escritura: calidad de líneas, tamaño de las letras, distribución de espacios del cuaderno, espacios entre letras y palabras, inversión, omisión y sustitución de letras, e incluso problemas para tomar el lápiz; muchos de ellos realizaban los trazos de abajo hacia arriba o escribían de derecha a izquierda. Lo que ninguno de los 20 participantes fue capaz de hacer se refiere a señalar los dibujos representativos de las palabras y enunciados copiados o a escribir palabras por dictado.

Para la segunda evaluación se observaron avances en prácticamente todos los alumnos del Grupo 1 (a excepción de los sujetos 4 y 9, que muestran el mismo nivel que en la primera evaluación); los alumnos 1,2 y 3 , que mostraron los mayores avances en lectura, son los que mostraron también mayores avances en escritura, logrando señalar correctamente los dibujos que representan las palabras y enunciados que copiaron, así como escribir números y palabras en dictado, identificando sus dibujos representativos; los demás alumnos de este grupo mostraron aproximaciones. Por el contrario, la mayoría de los alumnos del Grupo 2 (del 13 al 20) mantuvieron ejecuciones similares a las que obtuvieron en la primera evaluación, sólo los sujetos 11 y 12 lograron aproximaciones en identificación y dictado de palabras y números. La escritura de enunciados por dictado no fue realizada por ninguno de los 20 alumnos.

Para la tercera evaluación, cinco alumnos del Grupo 1 (sujetos 1, 2, 3, 4 y 7 ) y dos del Grupo 2 (12 y 14) realizaron prácticamente toda la prueba; mientras los sujetos 5 y 6 escribieron números y palabras por copia y dictado ubicando sus dibujos representativos, y escribieron enunciados por copia aunque mostraron errores en las pruebas de dictado de enunciados y en la comprensión de éstos; en el mismo caso estuvieron los sujetos 11, 13 y 15 , del Grupo 2. Los demás alumnos (tres del Grupo 1 y cinco del Grupo 2) continuaron mostrando muchos errores en prácticamente todas las habilidades de escritura que se evaluaron.

Al final del ciclo escolar, el nivel de escritura alcanzado es mejor en el Grupo 1. Siete de los alumnos de este grupo lograron realizar todas las habilidades de escritura, las excepciones fueron los sujetos 8,9 y 10 , que continuaron $\sin$ escribir enunciados por dictado y sin señalar dibujos representativos en enunciados copiados o dictados. En cambio, en el Grupo 2, sólo cuatro 
alumnos lograron contestar bien la prueba (sujetos del 11 al 14) y tres sujetos $(15,16$ y 17$)$ obtuvieron una ejecución similar a la alcanzada por los sujetos 8,9 y 10. Los últimos tres alumnos del Grupo 2 sólo pudieron copiar números, palabras y enunciados, fallando en las pruebas de comprensión de escritura, así como en todo el dictado. Además, la mayoría de los alumnos del Grupo 2 continuaron con problemas en la calidad de líneas, tamaño de las letras, distribución de espacios del cuaderno, espacios entre letras y palabras, así como inversión, omisión y sustitución de letras.

Las habilidades de composición fueron evaluadas a través de las pruebas cortas de administración frecuente, consistentes en presentar a los alumnos las diez palabras ya señaladas. El avance en estas habilidades fue más lento en todos los alumnos. Durante las dos primeras evaluaciones, los 20 niños se concretaron a copiar las palabras, algunos copiando correctamente y otros con los problemas de escritura ya reportados. Para la tercera aplicación de esta prueba, los alumnos con mejores ejecuciones en el IDEA fueron también los que comenzaron a escribir frases con las palabras presentadas, aunque ninguno elaboró una composición. Para el final del ciclo escolar, cinco niños del Grupo 1 realizan una composición (sujetos 1, 2, 3, 4 y 7) sin mostrar grandes problemas en la calidad de sus rasgos, tres de dichas composiciones fueron relativamente extensas y bien estructuradas; los otros cinco alumnos de este grupo continuaron copiando las diez palabras y mostraron algunos problemas de tamaño y calidad de letras, así como de distribución de espacios. En el Grupo 2, tres alumnos realizaron una composición y mostraron buena calidad de letra (sujetos 12, 13 y 14), pero la mayoría de los niños de este grupo presentó serios problemas de distribución de espacios, de calidad de líneas, así como de tamaño, sustitución e inversión de letras.

El análisis de los cuadernos de los alumnos, en los cuatro momentos de evaluación, permitió observar que las características de su escritura durante las aplicaciones de las evaluaciones realizadas (IDEA y elaboración de la composición), concordaban con las de los cuadernos que utilizaron durante sus clases y tareas escolares.

\section{Análisis del avance en matemáticas}

Durante la primera evaluación, todos los alumnos del Grupo 1, así como los sujetos 12, 13, 14, 16 y 19 del Grupo 2, fueron capaces de contar figuras, los demás contestaron correctamente sólo algunos de los reactivos; al preguntarles a los alumnos cuál conjunto tenía un número de figuras mayor o menor, sólo los sujetos 1,5 y 8 dieron todas las respuestas correctas, los demás alumnos tuvieron aproximaciones; ubicar las cifras correspondientes a un conjunto de figuras fue realizado por los sujetos $1,5,7$ y 8 , del Grupo 1 , así como por el 12 y el 19 del Grupo 2; encerrar en un círculo cierto nú- 
mero de unidades en conjuntos de figuras fue ejecutado correctamente por nueve alumnos del Grupo 1 (sujetos 1 al 9), así como por los sujetos 12, 13 y 19 , pero encerrar una decena de objetos sólo pudo hacerlo el sujeto 8 , este mismo alumno fue el único que pudo identificar signos de suma y resta. Otra habilidad que los alumnos mostraron fue sumar con figuras, los sujetos 1,2 , $3,5,7$ y 8 del Grupo 1 y los sujetos 12 y 19 del Grupo 2 lo hicieron correctamente en todos los casos, los demás alumnos mostraron algunas respuestas correctas; restar con figuras sólo lo hicieron correctamente los sujetos 1, 5, 7 y 8 del Grupo 1 y del Grupo 2 los sujetos 12, 13 y 19. Los reactivos relacionados con fracciones no fueron contestados correctamente por ningún niño, y tampoco los relativos a marcar en una cifra escrita el número correspondiente a las unidades o a las decenas; los alumnos no realizaron las sumas y restas escritas ni la solución de problemas que implican suma y resta.

Para la segunda aplicación de la prueba, sólo el sujeto 18 no pudo contar todas las figuras de manera correcta; el número mayor y menor de figuras fue ubicado por siete alumnos del Grupo 1 (sujetos 1, 2, 3, 5, 6, 8 y 9), así como por los sujetos 12 y 13; la mayoría de los alumnos pudo mencionar las cifras correspondientes al conjunto de figuras (exceptuando a los sujetos 4 y 7 , así como los sujetos 15,18 y 20 ); encerrar en un círculo cierto número de unidades en conjuntos de figuras fue ejecutado correctamente por nueve niños del Grupo 1 (el sujeto 4 no logró hacerlo), y por cinco niños del Grupo 2 (sujetos 11, 12, 13, 14 y 19); encerrar una decena de objetos fue realizado por seis y dos niños, respectivamente (sujetos $1,2,3,5,6$ y $8 ; 12$ y 13); siete alumnos del Grupo 1 (1, 2, 3, 5, 6, 8 y 9) y dos del Grupo 2 (12 y 13) identificaron correctamente signos de suma y resta; sumar con figuras ya fue realizado por nueve alumnos del Grupo 1 (exceptuando nuevamente al sujeto 4) y por siete alumnos del Grupo 2 (exceptuando nuevamente a los sujetos 15,18 y 20 ); restar con figuras fue logrado por siete y cinco niños, respectivamente $(1,2$, 3, 5, 6, 8 y 9 del Grupo 1; sujetos 11, 12, 13, 14 y 19, del Grupo 2). En esta segunda evaluación aparecen las primeras aproximaciones a la suma y resta con números de uno y dos dígitos, en la mayoría de los alumnos, pero son cinco (sujetos 1, 2, 3, 5 y 6 ) los que lo lograron para el caso de sumas, y tres (sujetos 2, 3 y 5) para el caso de restas y solución de problemas de suma y resta. Al igual que en la primera evaluación, ni los reactivos relacionados con fracciones ni los de marcar en una cifra escrita el número correspondiente a las unidades $o$ a las decenas fueron realizados.

En la tercera evaluación ya todos los alumnos pudieron contar figuras; el número mayor y menor de figuras fue ubicado por los mismos siete alumnos del Grupo 1, así como por los sujetos 11, 12 y 13; los sujetos 10, 18 y 20 continuaron sin poder mencionar las cifras correspondientes al conjunto de figuras, todos los demás lo hicieron; encerrar en un círculo cierto número de unidades en conjuntos de figuras fue ejecutado correctamente por los mis- 
mos nueve niños del Grupo 1 (el sujeto 4 no logró hacerlo), y por siete niños del Grupo 2 (los sujetos 10, 18 y 20 no lo realizaron); encerrar una decena de objetos fue realizado por los mismos seis niños del Grupo 1 que lo lograron en la segunda evaluación, y por tres del Grupo 2 (sujetos 1, 2, 3, 5, 6 y 8; 11, 12 y 13); nueve de los alumnos del Grupo 1 (el sujeto 4 no lo hizo) y cinco del Grupo $2(11,12,13,15$ y 19) identificaron correctamente signos de suma y resta; sumar con figuras ya fue realizado por todos los alumnos del Grupo 1 y por la mayoría del Grupo 2 (exceptuando nuevamente a los sujetos 18 y 20); restar con figuras fue logrado por ocho niños de cada grupo (exceptuando a los sujetos 4, 10, 18 y 20). En esta tercera evaluación se observan nuevos avances en la suma y resta con números de uno y dos dígitos, así como en la solución de problemas, pero quienes lo realizan correctamente fueron los sujetos 1, 2, 3, 5 y 6, del Grupo 1 y el sujeto 11 del Grupo 2. No se observan avances en lo relativo a fracciones ni en la ubicación del número correspondiente a las unidades o a las decenas en números escritos.

Para la cuarta evaluación se observan nuevos avances. Todos los alumnos realizaron correctamente casi todas las habilidades que implicaron figuras, fueron capaces de contarlas, de ubicar dónde había más y dónde menos, de mencionar las cifras correspondientes al conjunto de figuras y de encerrar en un círculo cierto número de unidades en conjuntos de figuras, aunque algunos fallaron al encerrar una decena de objetos (sujetos 7,9 y 10 , del Grupo 1, y sujetos 14, 16, 18 y 19 del Grupo 2). Los 20 alumnos lograron ubicar los signos de suma y resta, así como sumar y restar con figuras. Las habilidades para sumar y restar con números de uno y de dos dígitos, así como para la solución de problemas de suma y resta fueron logradas por la mayoría de los alumnos del Grupo 1 (sujetos 1, 2, 3, 4, 5, 6 y 8), los sujetos 7 y 9 resolvieron los problemas de suma y resta, así como las operaciones de un dígito, pero no las de dos dígitos, en tanto que el sujeto 10 se equivocó en todas estas habilidades. En lo que respecta al Grupo 2, sólo un alumno (sujeto 11) fue capaz de realizar las operaciones de uno y dos dígitos y de dar las respuestas correctas a los problemas de suma y resta, los sujetos 12 y 13 resolvieron algunos, pero no todos los problemas, y se equivocaron en las operaciones de dos dígitos; la mayoría de los alumnos del Grupo 2 (sujetos del 14 al 20) fallaron en todas estas habilidades.

Durante esta última evaluación, sólo el sujeto 11 fue capaz de ubicar números correspondientes a unidades y decenas en cifras escritas, los demás alumnos dijeron que esto no lo habían visto en clase, lo mismo ocurrió con los reactivos relativos a fracciones.

Una observación digna de resaltarse es el hecho de que, en todos los momentos de evaluación y en las tres áreas académicas, la mayoría de los niños de ambos grupos presentó problemas de excesiva tardanza, lo cual no afectó su puntaje en el IDEA ni el nivel de logro de las destrezas específicas 
evaluadas, pero sí parece ser indicativo de que sus habilidades académicas aún requieren un mayor grado de dominio.

\section{DISCUSIÓN}

El análisis de los aciertos y los errores a lo largo de las evaluaciones permitió conocer cómo se dio el avance y cuáles fueron los principales problemas en la adquisición de las distintas habilidades de lectura, escritura y matemáticas; la evaluación final permitió además, conocer cuáles objetivos curriculares fueron cubiertos por los alumnos y cuáles no lograron desarrollar.

Tomando en consideración todos los datos, puede concluirse lo siguiente, para la población aquí estudiada:

1. El nivel inicial de conducta, en las tres áreas académicas evaluadas, fue mayor en los niños que ingresaron con antecedentes de educación preescolar que en los que ingresaron sin dicha experiencia. Al parecer, los alumnos que cursan preescolar ingresan con mejores posibilidades de desarrollo académico.

2. Se observó un avance sostenido en la mayoría de los niños, en las tres áreas académicas, a lo largo del ciclo escolar, aunque los alumnos con experiencia preescolar mostraron siempre mejores ejecuciones y cubrieron un mayor número de objetivos educativos que los alumnos que ingresaron sin preescolar.

3. A pesar de lo anterior, para la cuarta evaluación la mayoría de los participantes de ambos grupos mostraron un desempeño por debajo del esperado, y no se cumplieron totalmente los objetivos primordiales de la lecto-escritura convencional y las matemáticas elementales.

Para el final del año escolar, sólo cuatro de los veinte niños evaluados fueron capaces de leer el cuento y contestar las preguntas relativas a su contenido. Es de llamar la atención que los problemas de fluidez, discriminación y comprensión siguieron presentes en la evaluación final de la lectura, en la mayoría de los niños evaluados, por lo que puede decirse que los objetivos del programa de lectura sólo fueron cubiertos parcialmente. En escritura ocurrió algo similar, nueve alumnos no lograron una ejecución adecuada en dictado y comprensión de enunciados, y la composición sólo fue realizada por ocho de los veinte alumnos evaluados, presentándose aún muchos problemas de calidad de escritura y excesiva tardanza en las ejecuciones. El área de matemáticas es donde se dieron menores diferencias entre grupos, y donde se logró cubrir un mayor número de los objetivos trabajados en clase, pero tampoco en este caso puede considerarse que los objetivos del curso se hayan 
cubierto cabalmente. En suma, podría hablarse de una falta de correspondencia entre las ejecuciones finales de los alumnos y las expectativas del programa de estudios, aún en el caso de los niños que ingresaron con preescolar, para las tres áreas académicas.

Por supuesto, los resultados de este estudio son esencialmente indicativos, pero contribuyen a argumentar acerca de las ventajas que le traería al sistema educativo mexicano la utilización de evaluaciones como las aquí reportadas, para analizar el proceso de adquisición de la lecto-escritura y las matemáticas en diferentes poblaciones escolares. Utilizar evaluaciones referidas a criterio, referidas al currículum y basadas en un análisis de tareas nos permite saber, no sólo el número de respuestas correctas e incorrectas -como ocurre con las pruebas de ejecución académica comúnmente utilizadas-, sino también conocer cuáles son los errores específicos que se están presentando en cada alumno, y por ende en cada grupo de estudiantes, para poder guiar la instrucción individual y grupal. La aplicación generalizada de este tipo de evaluaciones permitiría analizar los logros académicos en diferentes momentos del ciclo escolar, a fin de orientar los esfuerzos docentes hacia la consecución de los objetivos curriculares por parte de un mayor número de estudiantes. Para ello, sería útil acordar y formalizar un sistema general de evaluación del currículum escolar y de sus niveles de logro académico en toda la población estudiantil. Sólo de este modo podría decirse que el sistema educativo se nutre y enriquece de los hallazgos de la investigación educativa y de sus contribuciones.

También se requiere un análisis sistemático de las variables que puedan explicar por qué existe una falta de correspondencia entre las ejecuciones finales de los alumnos y las expectativas del programa de estudios, con datos que permitan comparar de manera clara los avances de diferentes poblaciones de alumnos, ubicados en distintos niveles académicos y pertenecientes a distintos niveles socioeconómicos y culturales.

Tomando como base los hallazgos del presente estudio, no es posible asegurar con certeza que los resultados académicos deficientes de los alumnos aquí estudiados guarden una estrecha relación con su nivel sociocultural de pertenencia, en virtud de que no se realizaron visitas a los hogares de los participantes, ni se estudió sistemáticamente el conjunto de variables que pudieran intervenir en este caso. Sin embargo, con base en lo reportado en la literatura disponible (Baker, et al, 2001; Buckner, et al, 2001; Carroll, et al, 2003; DiLalla, et al, 2004; Leppänen, et al, 2004; Muter, et al, 2004; Poe, et al, 2004), parece lógico suponer que dichos resultados se presentan en los participantes de este estudio por el hecho de pertenecer a un estrato socio cultural bajo, dado que se ha comprobado reiteradamente que las condiciones de pobreza se asocian comúnmente con un ambiente familiar que estimula poco las actividades y habilidades de tipo académico. Otros datos que apuntan en esa dirección los aporta el informe presentado por el Instituto Nacional 
para la Evaluación de la Educación (INEE, 2003) después de la aplicación de las pruebas nacionales a una muestra de 48 mil alumnos de sexto grado de primaria, pertenecientes a clases sociales diferentes. En dicho reporte se señala que, al comparar los puntajes de los alumnos tomando en consideración el contexto sociocultural de sus escuelas de pertenencia, en los contextos socioculturales desfavorables la gran mayoría de los alumnos obtuvo niveles considerados como "insatisfactorios"; la conclusión del INEE es que tales datos son indicadores de la fuerte influencia que tiene el "capital cultural" de las familias sobre el aprendizaje escolar, término en el que se incluyen variables como el nivel educativo materno y la existencia de bienes culturales como libros, materiales académicos y computadoras.

Independientemente del papel que juegan las familias en el desarrollo académico de los alumnos, los datos aquí reportados sugieren la necesidad de revisar qué tanto los programas de enseñanza preescolar estimulan los aspectos asociados a las áreas académicas a las cuales los niños estarán expuestos durante los primeros años de primaria. También se hace necesario analizar si las prácticas didácticas de los profesores, tanto a nivel preescolar como de primaria, resultan ser las adecuadas para promover el aprendizaje óptimo de los alumnos, tomando en cuenta su nivel de desarrollo. Otros aspectos que requieren ser estudiados sistemáticamente, por parte de la psicología educativa mexicana, se relacionan con los programas y actividades que se utilizan para la enseñanza de la lecto-escritura, las matemáticas y otras materias básicas a nivel primaria; es necesario conocer si en las aulas se llevan a cabo los procedimientos adecuados para promover el óptimo desarrollo académico de los alumnos.

En nuestra opinión, son muchas las contribuciones que la investigación psicológica general, y el modelo conductual en particular, pueden hacer a la educación en México, tanto en lo referente a la evaluación del proceso educativo, como en lo relativo a diseño curricular y manejo de factores relacionados con el bajo rendimiento escolar. Desafortunadamente, estos recursos teórico metodológicos no han sido lo suficientemente aprovechados en nuestro país. Se requiere que los psicólogos mexicanos contribuyan más a la investigación y a la solución de diversos aspectos de la problemática educativa nacional. Tal vez así se logre optimizar los recursos educativos y prevenir o disminuir los problemas que se presentan en un gran número de estudiantes, incluyendo los de aprendizaje y de rezago escolar. En cualquier caso, es importante que dicha investigación y práctica académica se realicen en los escenarios escolares, con los actores del proceso educativo (alumnos y profesores), y a través de la aplicación sistemática de los hallazgos teóricos y metodológicos de la psicología. 


\section{REFERENCIAS}

Adams, M. J., Treiman, R., \& Pressley, M. (1998). Reading, writing and literacy. En: W. Damon, I. E. Sigel, \& K. A. Renninger. (Eds.). Handbook of child psychology. Child psychology in practice. (Vol. 4) (pp. 275-355). New York: John Wiley \& Sons Inc.

Adelman, H. \& Taylor, L. (1993). Learning Problems and Learning Disabilities. California: Brooks/Cole.

Baker, L., Mackler, K., Sonnenschein, S. \& Serpell, R. (2001). Parents'interactions with their first-grade children during storybook reading and relations with subsequent home reading activity and reading achievement. Journal of School Psychology, $39,5,415-438$.

Bannan, B. \& Grabowski, B. (1995). The design of CD-I: Incorporating instructional design principles. Educational Technology, 35, 1, 36-39.

Becker, H.J. (1992). A model for improving the performance of integrated learning systems: Mixed individualized/group/whole class lessons, cooperative learning, and organizing time for teacherled remediation of small groups. Educational Technology, 32, 9, 6-15.

Bender, W. (1992). Learning Disabilities: characteristics, identification and teaching strategies. Boston: Allyn \& Bacon.

Biehler, R. y Snowman, J. (1992). Psicología Aplicada a la Enseñanza. México: Limusa.

Bowey, J. (1995). Socioeconomic status differences in preschool phonological sensitivity and first-grade reading achievement. Journal of Educational Psychology, 87, 3, 476-487.

Buckner, J. C., Bassuk, E. L. \& Weinreb, L. F. (2001). Predictors of academic achievement among homeless and low-income housed children. Journal of School Psychology, 39, 1, 45-69.

Carroll, J. M., Snowling, M. J., Hulme, C. \& Stevenson, J. (2003). The development of phonological awareness in preschool children. Developmental Psychology 39, 5, 913-923.

Dearing, E., McCartney, K., Weiss, H. B., Kreider, H. \& Simpkins, S. (2004). The promotive effects of family educational involvement for low-income children's literacy. Journal of School Psychology, 42, 6, 445-460.

De Jong, P. F. \& Leseman, P.M. (2001). Lasting effects of home literacy on reading achievement in school. Journal of School Psychology, 39, 5, 389-414.

DiLalla, L. F., Marcus, J. L. \& Wright-Phillips, M. V. (2004). Longitudinal effects of preschool behavioural styles on early adolescent school performance. Journal of School Psychology, 42, 5, 385-401.

Dockrell, J. \& McShane, J., (1993). Children's Learning Difficulties. Oxford"Blackwell Publishers.

Glazman, R., Figueroa, M., Follari, R. y Rodríguez, G. (1983). Corrientes Psicológicas y Currículum. En Una Década de la Facultad de Psicología: 1973-1983. Universidad Nacional Autónoma de México.

González, A. M. (2004). International perspectives of families, schools and communities: educational implications for family-school-community partnerships. International Journal of Educational Research, 41, 1, 3-9. 
Guevara, Y. (2006). Análisis interconductual de algunos elementos que constituyen la enseñanza básica. Revista Mexicana de Investigación Educativa, 11, 30, 10371064. (ISSN 1405-6666).

Guevara, Y. y Macotela, S. (2005). Escuela: del fracaso al éxito. Cómo lograrlo apoyándose en la psicología. México: Pax.

Hallahan, D. \& Kauffman, J. (1991). Exceptional Children: An Introduction to Special Education. (5a. Edición). New Jersey: Prentice Hall.

Hallahan, D., Kauffman, J. \& Lloyd, J.W. (1999). Introduction to Learning Disabilities (2a. Ed.) Boston: Allyn \& Bacon.

Hannafin, R. \& Freeman, D. (1995). An exploratory study of teachers views of knowledge adquisition. Educational Technology, 35, 1, 49-56.

Herman, J. \& Herman, J. (1995). Total quality management (TQM) for education. Educational Technology, 35, 3, 14-18.

Howell, K., Fox, S. \& Morehead, M. (1993). Curriculum-Based Evaluation: Tteaching and decision making. Pacific Grove. U.S.A.: Brooks/Cole.

Howell, K., Kaplan, S. \& O'Connell, L. (1979). Evaluating Exceptional Children. U.S.A.: Charles Merrill, Pub. Co.

Instituto Nacional para la Evaluación de la Educación. (2004). Resultados de las pruebas nacionales de aprovechamiento en lectura y matemáticas aplicadas al fin del ciclo 2002-2003. Dirección de Pruebas y Medición.

Kaufman, R. \& Swart, W. (1995). Beyond conventional benchmarking: integrating ideal visions, stategic planning, reengineering, and quality management. Educational Technology, 35, 3, 11-13.

Leppänen, U., Niemi, P., Aunola, K. \& Nurmi, J. E. (2004). Development of reading skills among preschool and primary school pupils. Reading Research Quarterly, 39, 1, 72-93.

López, F., Santoyo, C., Gómez, D. y Aguilar, J. (1995). Aprendizaje y desarrollo. En Mario Rueda (Ed.) Procesos de Enseñanza y Aprendizaje I. México: Consejo Mexicano de Investigación Educativa.

Macotela, S., Bermúdez, P. y Castañeda, I. (1991). Inventario de Ejecución Académica: Un modelo diagnóstico-prescriptivo para el manejo de problemas asociados a la lectura, la escritura y las matemáticas. Facultad de Psicología. Universidad Nacional Autónoma de México.

Macotela, S. (1993). Problemas de aprendizaje: Treinta años de debate. Facultad de Psicología. Universidad Nacional Autónoma de México.

Macotela, S., Bermúdez, P. y Castañeda, I. (1995). Evaluación de problemas de aprendizaje y bajo rendimiento. Facultad de Psicología. Cuaderno de prácticas integrales de Psicología Educativa. Universidad Nacional Autónoma de México.

Maldonado, A. (1999). Validación social de un instrumento referido a criterio. Tesis inédita de licenciatura en Psicología. Facultad de Psicología, Universidad Nacional Autónoma de México.

Martínez, R. (2002). Análisis del desempeño en la lecto-escritura y las matemáticas en una muestra de niños de primaria. Tesis inédita de licenciatura en Psicología. Facultad de Psicología, Universidad Nacional Autónoma de México.

Martínez, F., Cepeda, B., Fuentes, G. y Burgos, R. (1995). Evaluación del Aprendizaje. En Mario Rueda (Ed.) Procesos de Enseñanza y Aprendizaje II. México: Consejo Mexicano de Investigación Educativa. 
Martínez, R. A., Martínez, R. \& Pérez, M. H. (2004). Children's school assessment: Implications for family-school partnerships. International Journal of Educational Research, 41, 1, 24-39.

Morrison, E. F., Rimm-Kauffman, S. \& Pianta, R. C. (2003). A longitudinal study of mother-child interactions at school entry and social and academic outcomes in middle school. Journal of School Psychology, 41, 3,185-200.

Muter, V., Hulme, Ch., Snowling, M. J. \& Stevenson, J. (2004). Phonemes, rimes, vocabulary, and gramatical skills as foundations of early reading development: evidence from a longitudinal study. Developmental Psychology, 40, 5, 665-681.

Poe, M. D., Burchinal, M. R. \& Roberts, J. E. (2004). Early language and the development of children's reading skills. Journal of School Psychology, 42, 4, 315-332.

Resnick, L., Wang, M., \& Kaplan, J. (1973). Task Analysis and Curriculum Design: a hierarchical sequenced introductory mathematics curriculum. Journal of Applied Behavior Analysis, 6, 679-710.

Salsa, A. M. y Peralta, O. A. (2001). La "lectura" de material ilustrado: una estrategia educativa para el desarrollo del lenguaje de niños en edad preescolar. Revista Intercontinental de Psicología y Educación, III, 1, 49- 56.

Salvia, J. \& Hughes, Ch. (1990). Curriculum-Based Assessment. Testing what is taug$h t$. New York: MacMillan Publishing Co.

Spotts, T. \& Bowman, M. (1995). Faculty use of instructional technologies in higher education. Educational Technology, 35, 2, 56-64.

Strommen, E. (1992). Formative studies in the development of a new computer pointing device for young children. Educational Technology, 32, 4, 43-51.

Vacha, E. \& McLaughlin, T. (1992). The social structural, family, school, and personal characteristics of at-risk students: policy recommendations for school personnel. Journal of Education, 174, 3, 9-25.

Wallace, G., Larsen, S. \& Elksnin, L. (1992). Educational Assessment of Learning Problems. Austin: PRO-ED. 